Bundesgesundheitsbl -

Gesundheitsforsch - Gesundheitsschutz

2001 · 44:484-490 @ Springer-Verlag 2001
Leitthema: Sonnenschutz und Sonnenschutzmittel

J. Bertz $\cdot$ Robert Koch-Institut, Berlin

\section{Zusammenfassung}

Das maligne Melanom der Haut (MM) verursacht $90 \%$ der Todesfälle durch bösartige Neubildungen der Haut. 2\% aller auftretenden Krebskrankheiten in Deutschland sind MM. Die Patienten sind im Mittel etwa zehn Jahre jünger als Patienten mit anderen Krebskrankheiten. Die altersstandardisierten Erkrankungsraten von Männern und Frauen an MM haben sich in Deutschland - im Saarland und der DDR - seit Anfang der 70er Jahre bis Ende der 80er Jahre auf 7 bis 8 pro 100000 im Europastandard mindestens verdoppelt. Diese Entwicklung fand in ähnlichem Maße in allen Ländern Europas und in Ländern mit Bevölkerungen vorwiegend europäischen Ursprungs statt. Im Unterschied dazu ist die Sterblichkeit an MM in weit geringerem Maß angestiegen. Bei Frauen blieb der erwartete Anstieg der altersstandardisierten Sterberaten nahezu vollkommen aus. Frauen weisen darüber hinaus bei nahezu gleicher Höhe der Erkrankungsraten um ein Drittel niedrigere Sterberaten als Männer auf.

\section{Schlüsselwörter}

Melanom der Haut · Inzidenz . Mortalität · Überlebensraten · Risikofaktoren . Prävention

\title{
Epidemiologie des malignen Melanoms der Haut (ICD'9: 172)
}

M aligne Melanome sind bösartige Neubildungen der Pigmentzellen, der Melanozyten. Die weit überwiegende Zahl dieser Neubildungen entsteht im Bereich der Haut. Darüber hinaus kommen an den Schleimhäuten, zum Beispiel des Darms, an der Netz- und Aderhaut des Auges sowie an den Hirnhäuten maligne Melanome vor. Etwa 90\% aller Todesfälle an Hautkrebs gehen auf maligne Melanome (MM) zurück. Nach einer Schätzung des Robert Koch-Instituts für das Jahr 1997 erkranken in Deutschland jährlich 3100 Männer und 3800 Frauen an MM.140o dieser Erkrankungsfälle bei Männern und 1500 Erkrankungen bei Frauen treten bereits vor dem 6o. Lebensjahr auf. Damit macht das MM insgesamt etwa $2 \%$ aller auftretenden Krebskrankheiten aus, im Alter unter 60 Jahren jedoch mehr als 3\%. Das mittlere Erkrankungsalter ergibt sich für beide Geschlechter mit etwa 56 Jahren deutlich niedriger als für andere Krebskrankheiten. Zum Vergleich: Für Krebs insgesamt liegt es bei 65,5 für Männer und über 67 Jahren für Frauen [1].

\section{Klinik}

Klinisch und histologisch lassen sich im wesentlichen vier verschiedene Melanomtypen voneinander unterscheiden:

Das superfiziell spreitende Melanom (SSM) beginnt mit einer horizontalen Ausbreitungsphase zunächst als Fleck, entwickelt sich erst dann invasiv flach erhaben, häufig mit farblichen Unregelmäßigkeiten und sekundär knotigen Anteilen.
Das noduläre Melanom (NM) imponiert hingegen als primär knotiger, erhabener, überwiegend schwarzbrauner Tumor, dem eine anfänglich horizontale Wachstumsphase und damit die Möglichkeit zur Frühdiagnose zu fehlen scheint. Beide erstgenannten MM-Typen treten bereits in jüngerem Alter auf.

Das Lentigo-maligna-Melanom (LMM) entsteht oft erst nach vielen Jahren aus einer Lentigo maligna nahezu ausschließlich im Gesichtsbereich älterer Patienten.

Das akral-lentiginöse (akrolentiginöse) Melanom (ALM) findet sich vorwiegend an Handtellern und Fußsohlen, aber auch um und unter Fuß- und Fingernägeln [2] (Tabelle 1).

Die Tumortypen unterscheiden sich deutlich nach der Tumordicke, die in Millimetern nach Breslow [3] am Operationspräparat bestimmt wird. Die dicksten, damit prognostisch ungünstigsten Tumore sind im Mittel die NM mit einem Median der Tumordicke von 2,5 mm. SSM und LMM erwiesen sich gleichermaßen als prognostisch günstige Tumoren mit einem Median der Tumordicke von $0,70 \mathrm{~mm}$ beim LMM und o,80 mm beim SSM. Das ALM nahm mit einem Median der Tumordicke von 1,4 $\mathrm{mm}$ eine Zwischenstellung ein (Tabelle 1).

Die bevorzugte Lokalisation des ALM ist bei Männern wie Frauen quasi namengebend - an den Akren, insbe-

Dr. Joachim Bertz

Robert Koch-Institut, Nordufer 20,13352 Berlin 


\section{J. Bertz}

\section{Cutaneous Melanoma in Germany}

\begin{abstract}
$90 \%$ of all skin cancer death in Germany is caused by malignant Melanoma (MM). MM is responsible for about $2 \%$ of all cancer cases diagnosed in Germany. On average Melanoma-patients are about 10 years younger than other cancer-patients. The age standardized incidence rates of males and females at least doubled in the German cancer registries of the Saarland and the German Democratic Republic in the 20 years during the seventies and eighties, reaching 7-8 per 100,000 European standard-population. Similar increases have been observed in other European countries as well as in countries with populations of mostly European origin outside Europe. In contrast to incidence the increase of age standardized mortality rates is less steep. For females there is nearly no increase of mortality at all. Furthermore, females have mortality rates, which are about one third lower than those of males.
\end{abstract}

\section{Keywords}

Melanoma of the skin · Incidence · Mortality · Survival $\cdot$ Risk factors $\cdot$ Prevention

Tabelle 1

Histologische Subtypen des Melanoms

\begin{tabular}{lcll} 
MM-Typ & $\begin{array}{l}\text { Anteil } \\
(\%)\end{array}$ & $\begin{array}{l}\text { Alters- } \\
\text { median } \\
\text { (Jahre) }\end{array}$ & $\begin{array}{l}\text { Tumordicken- } \\
\text { median (mm) }\end{array}$ \\
\hline SSM, superfiziell spreitend & 58 & 51 & 0,8 \\
NM, nodulär & 21 & 56 & 2,5 \\
LMM, Lentigo-maligna & 9 & 68 & 0,7 \\
ALM, akral-lentiginöses & 4 & 63 & 1,4 \\
\hline
\end{tabular}

Ergebnisse des Zentralregisters Malignes Melanom 1983-1995 ( $n=30015)$

sondere an Hand- und Fußsohlen, Finger- und Zehennägeln. Gleichermaßen bei Männern und Frauen finden sich LMM am häufigsten im Kopf-Halsbereich älterer Menschen (Männer 55\%, Frauen 64\%). SSM und NM weisen ein nahezu gleiches Lokalisationsmuster auf, das von dem der ALM und LMM stark abweicht. Die häufigste Lokalisation beim SSM und NM, die zusammen $80 \%$ aller Melanome der Haut repräsentieren, ist bei Männern der Rumpf (SSM $64 \%$, NM 53\%), insbesondere der Rücken, und bei Frauen die unteren Extremität (SSM 48\%, NM 44\%), besonders die Unterschenkel.

\section{Prognose und Therapie}

Ca. $90 \%$ aller Melanompatienten in dermatologischen Kliniken kommen derzeit nach Daten des Zentralregisters Malignes Melanom ohne erkennbare Metastasierung zur ersten Diagnose. Der wichtigste prognostische Faktor beim primären MM ohne Metastasen ist die vertikale Tumordicke nach Breslow [3], die am histologischen Präparat bestimmt wird (bis unter $0,75 \mathrm{~mm}$ : ca. $97 \%$ Zehnjahres-Überlebensrate; 0,76 bis unter $1,5 \mathrm{~mm}$ : ca. $90 \%$; 1,5 bis unter $4 \mathrm{~mm}$ ca. $65 \%$; größer als $4 \mathrm{~mm}$ : ca. 50\%). Andere Indikatoren wie der Invasionslevel nach Clark [4], der klinisch-histologische Typ (ungünstig: primär noduläre Melanome und akrolentiginöse Melanome), das Geschlecht (schlechtere Prognose für Männer) und die Tumorlokalisation (ungünstigere Prognose für oberen Stamm, Oberarme, Hals und behaarten Kopf) haben vergleichsweise geringe Relevanz, sobald die Tumordicke berücksichtigt wird $[5,6]$.
Das MM kann sowohl über die Lymphals auch über die Blutbahnen metastasieren. Etwa zwei Drittel aller Erstmetastasierungen sind zunächst auf das regionäre Lymphabflussgebiet beschränkt. Die Zehnjahres-Überlebensrate beträgt bei Patienten mit Satelliten- und Intransit-Metastasen ca. 25-40\% und bei Patienten mit klinisch manifesten regionären Lymphknoten-Metastasen ca. 15-30\%. Eine bereits erfolgte Fernmetastasierung verschlechtert die Prognose so, dass ohne Behandlung eine mediane Überlebenszeit von ca. vier bis sechs Monaten resultiert [2].

\section{"Der wichtigste prognostische Faktor beim malignen Melanom ohne Metastasen ist die vertikale Tumordicke."}

Die primäre Therapie eines klinisch gesicherten MM ist immer die vollständige operative Entfernung mit einem Sicherheitsabstand von $1 \mathrm{~cm}$. Die Operation erfolgt inzwischen meist in Lokalanästhesie. Dieses Vorgehen spiegelt den Trend zu kleineren Sicherheitsabständen wider, der international zu beobachten ist [7]. In Abhängigkeit von dem histopathologischen Befund des Operationspräparats kann im Rahmen einer Nachexzision ein größerer Sicherheitsabstand erreicht werden. Damit kann vor allem ein lokales Rezidiv verhindert werden. Lymphknotenpakete werden nur noch bei Befall der ersten Lymphknotenstation nach dem Befund der selektiven Lymphknotenbiopsie entfernt. Insgesamt hat die Radikalität der Erstoperation in letzter Zeit abgenommen und einem schonenderen Verfahren Platz gemacht. Nur bei schlechter Prognose, d. h. 
großer Tumordicke oder bereits erfolgter Metastasierung, bietet eine zusätzliche so genannte adjuvante Therapie z. B. mit Interferonen einen Vorteil. Kurative Erfolge sind in diesem Stadium allerdings selten. Strahlen- und Chemotherapie (auch als hypertherme Perfusionstherapie bei Extremitätensitz) sind nur lokal und damit palliativ wirksam [2].

\section{Inzidenz und Mortalität}

Die deutschen Sterberaten an MM der Haut und die entsprechenden Erkrankungsraten im Saarland, dem einzigen deutschen Krebsregister, das seit 1970 kontinuierlich und vollständig Erkrankungsdaten dokumentiert, liegen im Vergleich zu anderen europäischen Ländern in einem mittleren Bereich. Die höchsten altersstandardisierten Erkrankungsraten werden in Schweden, Dänemark und den Niederlanden beobachtet, die niedrigsten in Griechenland, Portugal und Spanien [1]. Das Vorkommen des MM in Europa scheint in weit stärkerem Maße von konstitutionellen Merkmalen der Pigmentierung beeinflusst als von Unterschieden der Sonnenexposition.

\section{„Das Vorkommen des malignen Melanoms in Europa ist stark von der konstitutionellen Pigmentierung beeinflusst."}

Die altersstandardisierte Inzidenz an MM der Haut ist zwischen 1970 und 1990 bei Männern wie bei Frauen im Saarland wie in der DDR erheblich angestiegen. Für den Zeitraum zwischen Mitte der 6oer und Mitte der 8oer Jahre ließ sich in europäischen Krebsregistern und unter Bevölkerungen europäischen Ursprungs in den USA und Kanada im Mittel ein jährlicher Anstieg der altersstandardisierten Erkrankungsraten von $3 \%$ bis $7 \%$ feststellen [7]. Die Erkrankungsraten im ehemals nationalen Krebsregister der DDR stiegen während dessen um 3,5\% bei Frauen und 3,8\% bei Männern. Mindestens in gleichem Maße sind die saarländischen Erkrankungsraten angestiegen (Abb. 1). Der weitere Verlauf der Erkrankungsraten nach 1990 im Saarland zeigt das Ende dieser Entwicklung an. Innerhalb von 20 Jahren haben die Erkrankungsraten sich mindestens verdoppelt. Sämtliche Altersbereiche nehmen am Anstieg der Erkrankungshäu- figkeit teil. Während des gesamten Zeitraums werden die altersstandardisierten Erkrankungsraten der Frauen im Saarland und der DDR ein wenig höher gemessen als die der Männer. In Zehnjahresaltersgruppen ist ein exakterer Vergleich der Erkrankungshäufigkeit von Männern und Frauen im Saarland möglich. Über zehn Kalenderjahre gemittelt ergeben sich höhere altersspezifische Erkrankungsraten der Frauen bis zum Alter von 50, nahe$\mathrm{zu}$ gleich hohe Erkrankungsraten von Männern und Frauen zwischen 50 und 60 Jahren, gefolgt von höheren Erkrankungsraten für über 6ojährige Männer (Abb. 2). Das führt im Ergebnis zu ähnlich hohen altersstandardisierten Erkrankungsraten von Männern und Frauen.

Deutlichere Unterschiede zwischen Männern und Frauen ergeben sich bei den altersstandardisierten Sterberaten in den alten und neuen Bundesländern (Abb.3).Die Sterberaten der Männer sind im Osten wie im Westen jeweils deutlich höher als die der Frauen. Die altersstandardisierten Mortalitätsraten der Männer steigen bis zu den frühen goer Jahren in den alten und neuen Bundesländern geringfügiger an als die Erkrankungsraten. Die Sterberaten der Frauen verbleiben nach anfänglich flacherem Anstieg im Osten und Westen Deutschlands bereits mit Ende der 8oer Jahre nahezu unverändert. Ein flacherer Anstieg der altersstandardisierten Sterberaten an MM im Vergleich zum Anstieg der Erkrankungsra- ten, ein flacherer Anstieg der Sterberaten der Frauen im Vergleich zu denen der Männer und höhere Sterberaten der Männer sind in verschiedenen europäischen Ländern und unter Bevölkerungen europäischen Ursprungs in den USA, Kanada und Neuseeland zu beobachten [7]. Das frühere Einschwenken oder Abflachen der Sterbraten der Frauen konnte für die USA und Dänemark bereits in den 8 oer Jahren beobachtet werden.

\section{„Die Sterberaten der Männer sind im Osten wie im Westen jeweils deutlich höher als die der Frauen."}

Die Darstellung der altersstandardisierten Sterberaten wird insbesondere bei Männern der neuen Bundesländer durch einen Anstieg der Raten zwischen 1990 und 1992 gestört, der lediglich auf die Anpassung der Todesursachenstatistik im Zuge der Vereinigung zurückgeht (Abb.3). Von solchen störenden Strukturbrüchen unbeeinflusst bleibt die altersspezifische Sterblichkeit an MM für Frauen (Abb. 4) und Männer (Abb. 5) in den alten Bundesländern. Die Sterblichkeit der Frauen ist in allen Altersbereichen deutlich geringer als die der Männer. Für Männer wie Frauen gilt: Je jünger die Altersgruppe, desto früher zeichnet sich ein Ende des Anstieges der Sterberaten ab, dem ein Rückgang folgt. Für Frauen ist dies jeweils etwas frü-

\section{Altersstandardisierte Erkrankungsraten}

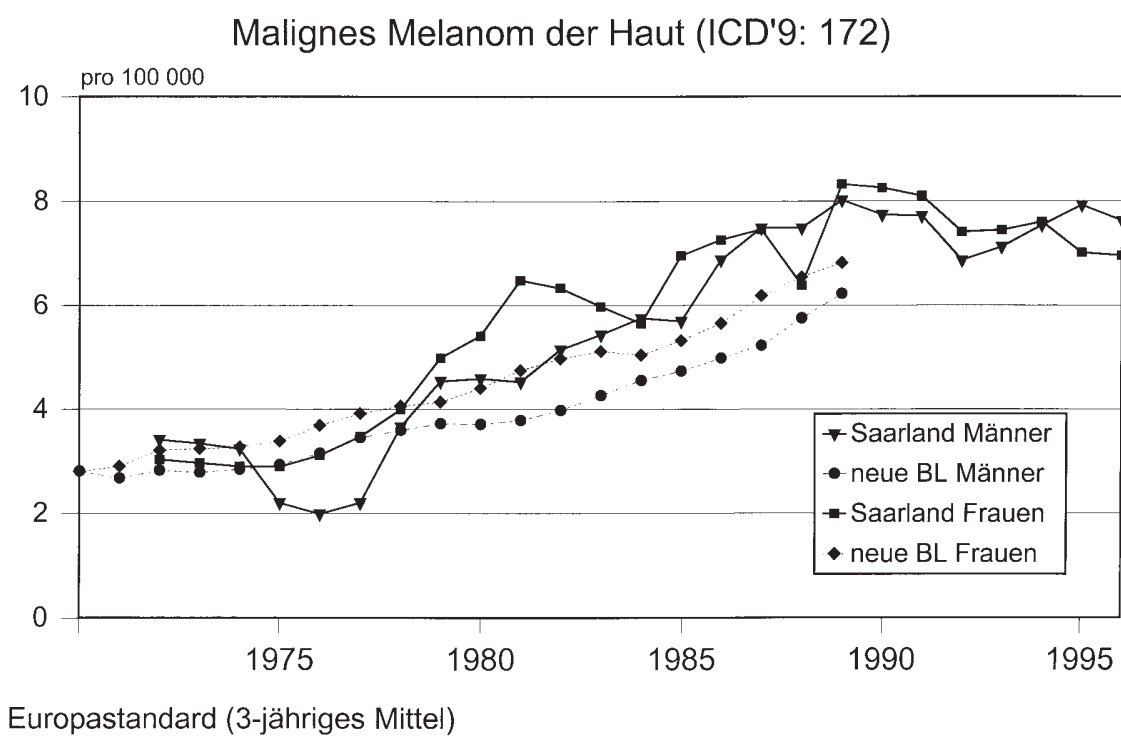

Abb. $1 \triangle$ Verlauf der altersstandardisierten Erkrankungsraten an MM der Haut Saarland und DDR 


\section{Altersspezifische Erkrankungsraten}

Malignes Melanom der Haut (ICD'9: 172)

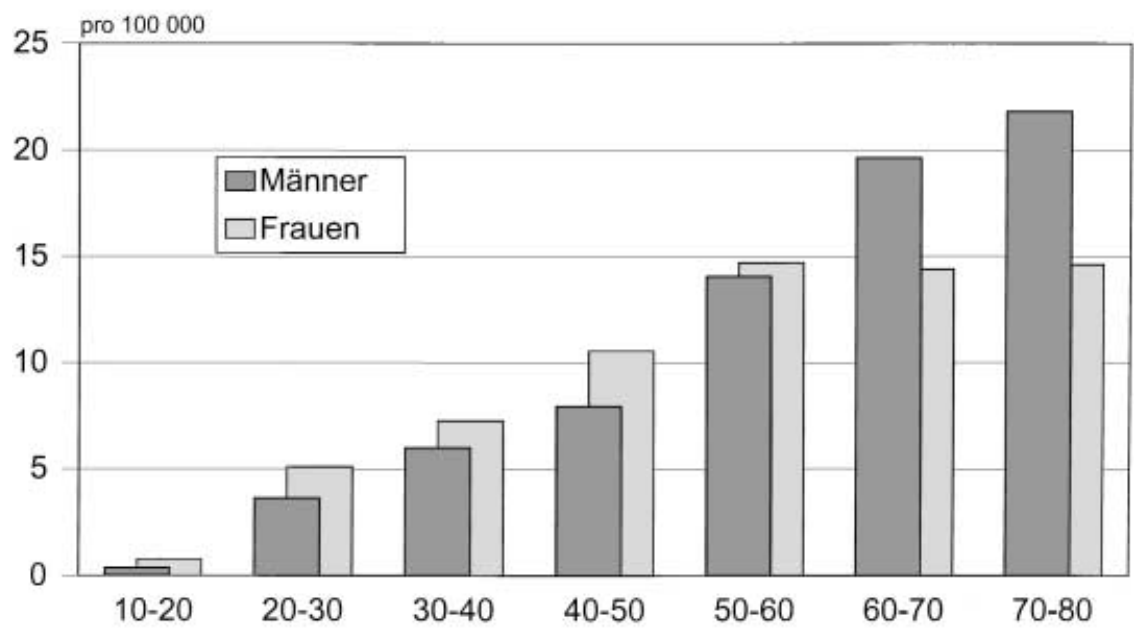

Krebsregister des Saarlands 1987-1996

Abb.2 A Altersverteilung der altersspezifischen Erkrankungsraten an MM der Haut Saarland 1987-1996

her als für Männer zu beobachten. Während die Sterberaten 60- bis 70-jähriger Frauen bereits rückläufig sind, nehmen die Sterberaten dieser Altersgruppe bei Männern noch geringfügig zu. Bei Männern und bei Frauen der alten Bundesländer nehmen die Sterberaten über 70-Jähriger noch zu und die Sterberaten unter 60-Jähriger bereits ab. In den 6oer und 7oer Jahren ergab sich für dänische, britische, kanadische und US-amerikanische Sterberaten an MM ein ähnliches Bild. Damals war der Anstieg der Sterberaten unter 55-Jähriger bereits abgeflacht oder hatte sich sogar in einen leichten Rückgang gewandelt, während die Sterberaten Älterer noch weiter anstiegen. In neun von 38 Datensätzen zur Inzidenz und in 13 von 18 Datensätzen zur Entwicklung der Mortalität an MM in Ländern mit Bevölkerungen europäischen Ursprungs kam es zu einer vergleichbaren Entwicklung [7]. Diese Konstellation der altersspezifischen Sterberaten deutet auf einen Geburtskohorteneffekt hin.

\section{„Der Geburtskohorteneffekt macht deutlich, dass das MM-Risiko wahrscheinlich relativ früh im Lebenslauf festgelegt wird und dann stabil über ein ganzes Leben bestehen bleibt."}

Auswertungen von Sterbedaten aus Kanada, USA und Großbritannien zeigen einen mit den Geburtsjahrgängen zwischen 1890 und 1905 beginnenden Anstieg des MM-Risikos, der mit den Geburtsjahrgängen zwischen 1925 und 1935 beendet ist [8]. Die beschriebenen Besonderheiten der deutschen altersspezifischen Sterberaten stimmen mit einem derartigen Geburtskohorteneffekt gut
Europastandard überein. Die auch bis in die 9oer Jahre noch ansteigenden Sterberaten der über 70-Jährigen repräsentieren gerade die Geburtsjahrgänge zwischen 1925 und 1935. Das würde bedeuten, dass eine weitere Zunahme der Sterblichkeit an MM nicht mehr zu befürchten ist. Der Geburtskohorteneffekt macht deutlich, dass das MM-Risiko wahrscheinlich relativ früh im Lebenslauf festgelegt wird und dann stabil über ein ganzes Leben bestehen bleibt.

\section{Überlebensraten}

Daten des saarländischen Krebsregisters und des nationalen Krebsregisters der DDR zeigen erhebliche Verbesserungen der Überlebensraten mit der Zeit, wie sie auch international beobachtet werden. Zeitgleich mit der Zunahme der Inzidenz vor allem zwischen 1970 und 1985 kommt es nicht nur zu einer bloße Vorverlagerung der Diagnosestellung. Vielmehr verbessern sich die FünfjahresÜberlebensraten deutlicher als die Einund Zweijahres-Überlebensraten. Dem entspricht ein zunehmend größerer Anteil von zuletzt $80 \%$ der Patientinnen und $69 \%$ der Patienten im Saarland, die nicht an ihrer Krebserkrankung versterben und insofern als „geheilt" bezeichnet werden können [9]. Im Vergleich zur Allgemeinbevölkerung gehen Anfang der 7oer Jahre im Saarland den Patienten noch $48 \%$, den Patientinnen $39 \%$ ih-

\section{Altersstandardisierte Sterberaten}

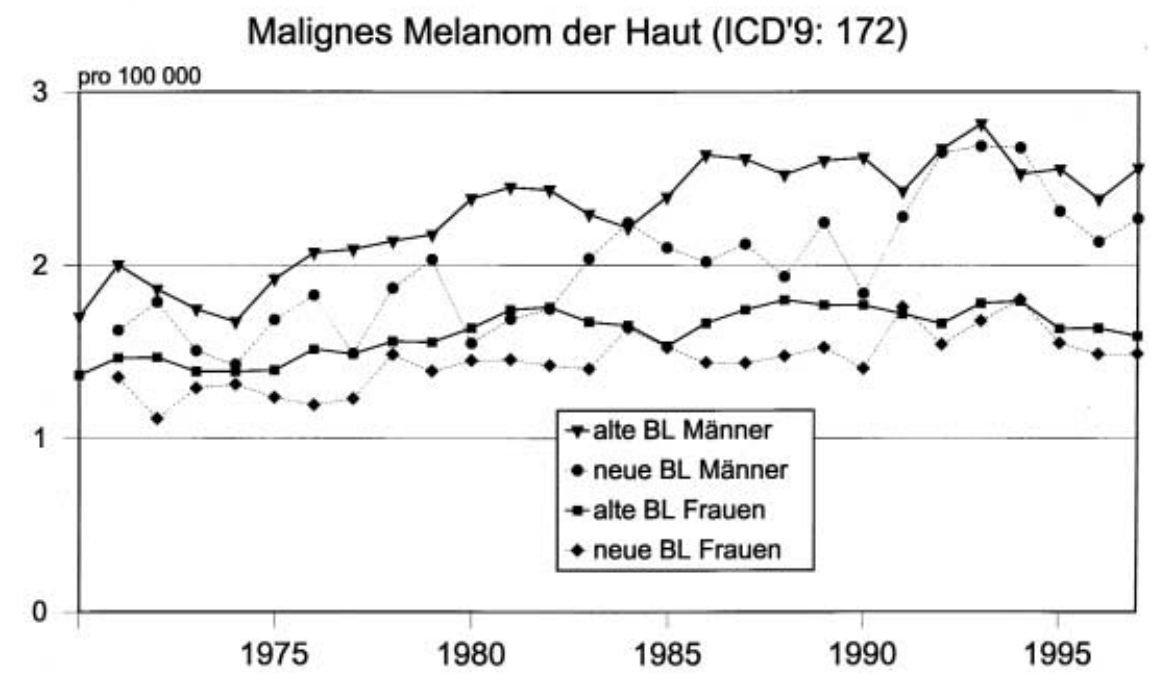

Abb.3 Verlauf der altersstandardisierten Sterberaten an MM der Haut - alte und neue Bundesländer 


\section{Altersspezifische Sterberaten Frauen}

Malignes Melanom der Haut (ICD'9: 172)

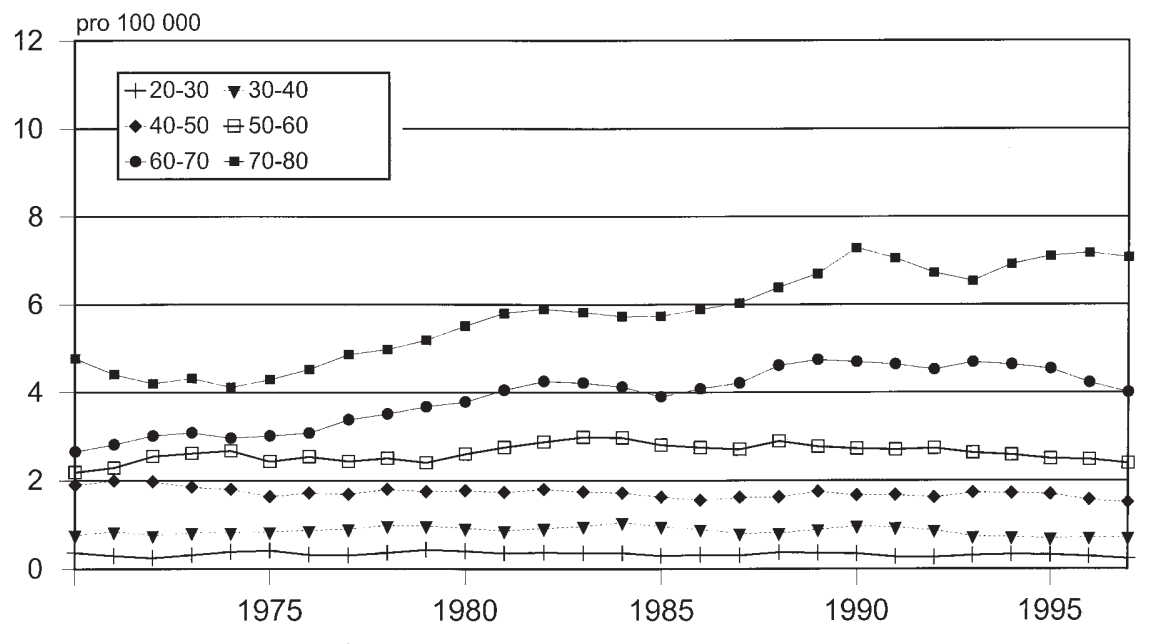

Alte BL (3-jähriges Mittel)

Abb.4 Verlauf der altersspezifischen Sterberaten der Frauen an MM der Haut - alte und neue Bundesländer rer ferneren Lebenserwartung durch den frühen Tod an MM verloren. Zuletzt gehen den Männern noch $20 \%$, den Frauen $14 \%$ ihrer ausstehenden Lebensjahre verloren [9]. Ursache für diesen Überlebensvorteil von Frauen dürfte ein größerer Anteil dünner MM sein. Vieleinsetzenden gesetzlichen Früherkennung eine größere Aufmerksamkeit gegenüber Hautveränderungen zu frühzeitigeren Diagnosen bei Frauen bei. Im Verlauf der 8oer Jahre konnte mit Hilfe des Zentralregisters Malignes Melanom der Deutschen Dermatologischen Gesellschaft gezeigt werden, dass MM der Haut zunehmend in früheren Stadien, das heißt dünner, diagnostiziert wurden. Im Vergleich der Zeitintervalle 1983-1986 zu 1987-199o kam es bei beiden Geschlechtern zu einer signifikanten Abnahme der Tumordicke. Bei Männer nahm der Anteil an dünnen Tumoren mit einer Tumordicke $<0,75 \mathrm{~mm}$ im Zeitraum $1983-1986$ von $31 \%$ auf $38 \%$ im Zeitraum 1987-1990 zu, während er sich bei Frauen in den genannten Zeiträumen sogar von $35 \%$ auf $44 \%$ erhöhte [10]. Zwar nahm der Anteil dünnerer MM auch bei Männern zu, die MM der Männer blieben jedoch im Mittel dicker als die der Frauen. Diesen Rückstand bei der Früherkennung gilt es aus Sicht der Männer aufzuholen. leicht trägt neben der bei Frauen früher

\section{Risikofaktoren}

Das Risiko am MM der Haut zu erkranken, hängt einerseits von konstitutionellen, andererseits von äußeren Faktoren ab. Da die SSM und NM alle anderen histologisch unterscheidbaren Subtypen weit überwiegen - zumal in jüngerem Lebensalter - beziehen sich die meisten
Risikostudien auf diese beiden Typen des MM der Haut, die hauptsächlich an gewöhnlich bedeckten Körperpartien auftreten. Bezüglich der konstitutionellen Faktoren ergibt sich aus diesen epidemiologischen Studien ein breites Spektrum risikomindernder wie risikoerhöhender Pigmentierungscharakteristika. Zu nennen sind zunächst Hautfarbe (unter der Achsel), Augen- und Haarfarbe (am Ende der Pubertät). Hellhäutige, insbesondere solche mit Sommersprossen, Blau- oder Grünäugige, Rotblonde und Blonde tragen das größere Risiko. Asiaten und Afrikaner erkranken dagegen nur in den seltensten Fällen am MM der Haut. Betroffen sind dann nahezu ausschließlich die geringer pigmentierten Handteller und Fußsohlen. Ein weiterer Risikofaktor ist der Hauttyp, der sich aus der Reaktion der Haut auf Sonneneinstrahlung ableitet. Wer auf Sonneneinstrahlung nie mit Sonnenbrand, sondern immer mit Sonnenbräune reagiert, hat das niedrigste MM-Risiko. Wer immer mit Sonnenbrand und nie mit Bräune reagiert, hat den ungünstigsten Hauttyp [11]. Noch wichtiger als diese konstitutionellen Risikofaktoren ist die Zahl der erworbenen Pigmentmale (Nävuszell-Nävi). Dies gilt nicht nur für das Extrem des familial atypical multiple mole melanoma syndrome (FAMMM), das über polygene Erbfaktoren zur familiären Häufung führen kann

\section{Altersspezifische Sterberaten Männer}

\section{Malignes Melanom der Haut (ICD'9: 172)}

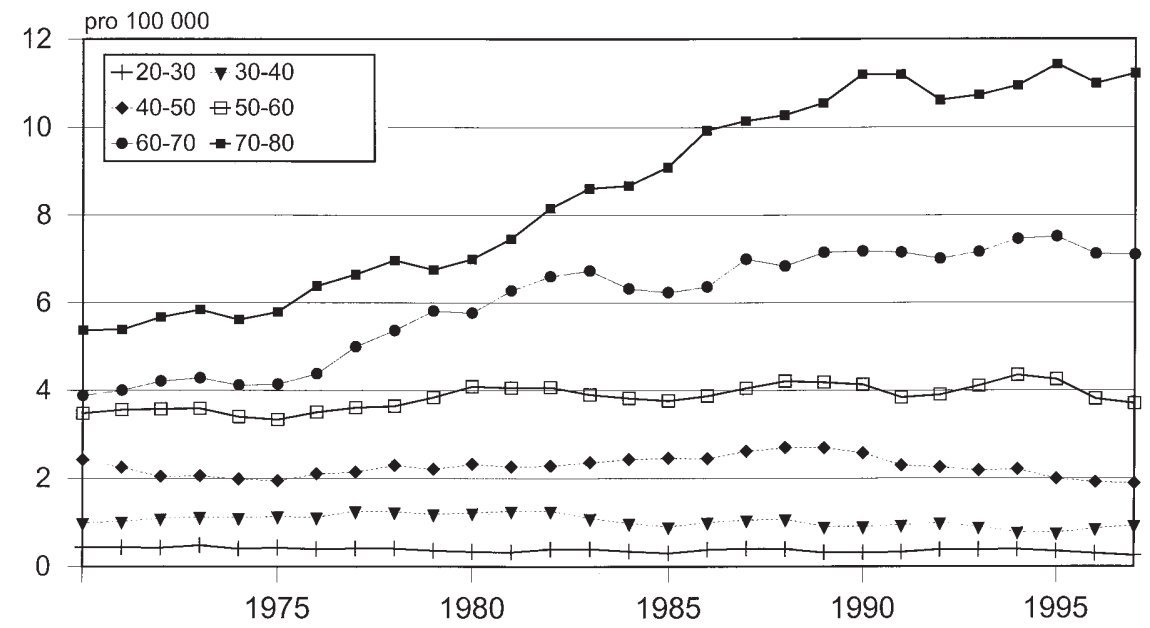

Alte BL (3-jähriges Mittel)

Abb. 5 Verlauf der altersspezifischen Sterberaten der Männer an MM der Haut - alte und neue Bundesländer 
[12]. Die im Volksmund „Muttermale“ genannten Pigmentmale sind zur weit überwiegenden Zahl nicht angeboren, wie der Name suggeriert, sondern werden im Laufe der Kindheit erworben. Möglicherweise handelt es sich bei einem Teil dieser Nävi um Vorläufer des MM. Unabhängig davon erklärt die Anzahl dieser hauptsächlich in der Kindheit erworbenen Pigmentmale den größten Teil des MM-Risikos [11, 12, 13]. Im Unterschied zu den zuerst genannten konstitutionellen Risikofaktoren ist die Zahl der Pigmentmale ein durch äußere Faktoren beeinflussbarer konstitutioneller Risikofaktor.

\section{„Intensive Sonnenexposition in der Kindheit bestimmt das spätere Risiko, an einem mali- gnen Melanom zu erkranken."}

Epidemiologische Studien an Migranten in den USA und Australien haben gezeigt, dass intensive Sonnenexposition in der Kindheit das spätere MM-Risiko bestimmt. Nur Hellhäutige, die noch im Alter unter zehn Jahren aus weniger sonnenverwöhnten Staaten der USA nach Kalifornien oder von den britischen Inseln nach Australien einwanderten, erreichen in ihrem späteren Leben nahezu das hohe MM-Risiko der dort bereits geborenen Hellhäutigen. Eine spätere Einwanderung im Alter von über 20 Jahren führt dagegen zu einem deutlich niedrigeren MM-Risiko, nicht wesentlich höher als im Herkunftsstaat bzw. auf den britischen Inseln [11]. Die Intensität der Sonneneinstrahlung, der Sonnenexposition, in der Kindheit fördert die Zunahme von Pigmentmalen und führt so zu einem höheren MM-Risiko im späteren Leben $[12,13,14]$. Die Zahl schmerzhafter Sonnenbrände kann als gut erinnerbares Maß für die Intensität intermittierender Sonnenbestrahlung in der Freizeit gelten. Das MM-Risiko steigt mit der Zahl der Sonnenbrände. Es ist sicher, dass intensive Sonnenexposition in der Kindheit das MM-Risiko erhöht. Weniger klar sind die Folgen der Freizeit-Sonnenexposition im Erwachsenenalter. Möglicherweise tragen auch Sonnenbrände im Erwachsenenalter zur Entwicklung von MM an üblicherweise bedeckten Körperpartien bei [13, 15]. Bei Männern ist davon am häufigsten der Rücken, bei Frauen die untere Extremität betroffen.
Etwas anders stellt sich der Zusammenhang zwischen Sonnenexposition und Lentigo-malignem Melanom dar. Das höhere Alter der Patienten und die bevorzugte Lokalisation der LMM (Lentigo-malignen Melanome) im Gesicht sprechen dafür, dass die LMM in ähnlicher Weise wie die nicht melanotischen Hauttumoren, Basaliom und Spinaliom, durch chronische, kumulative Sonnenexposition gefördert werden [11].

\section{Prävention}

Zur Prävention des MM bieten sich Maßnahmen der primären wie der sekundären Prävention an. Die primäre Prävention soll die Entstehung von MM verhindern. Die Entwicklung von MM wird im Erwachsenenalter möglicherweise durch intensive intermittierende Sonneneinstrahlung auf blasse Haut gefördert. Sicher ist jedoch, dass durch eine derartige intensive Freizeit-Sonnenexposition im Kindes- und Jugendalter das MM-Risiko im späteren Erwachsenenalter erhöht wird. Die einfachste Maßnahme zur Senkung des MM-Risikos Hellhäutiger wäre eine vorsichtigere Bräunung im Halbschatten und eine Mittagspause im Schatten. Zumindest Kindern sollte klar gemacht werden, dass Sonnenbrände kein Königsweg zur Sonnenbräune, sondern ein ernst zu nehmender Unfall auf dem Weg dorthin sind.

\section{"Durch die Lokalisation auf der Körperoberfläche eignet sich das maligne Melanom der Haut besonders zur Früherkennung."}

Die sekundäre Prävention des MM soll zur frühzeitigen Diagnose weniger weit fortgeschrittener, d. h. dünnerer MM führen, deren Therapie noch mit guten bis sehr guten Aussichten auf Heilung verknüpft ist. Durch die Lokalisation auf der Körperoberfläche erscheint das MM der Haut besonders zur Früherkennung geeignet [16]. Für Frauen schließt das Krebsfrüherkennungsprogramm der gesetzlichen Krankenkassen ab dem 30. Lebensjahr Fragen zu Hautveränderungen ein, die der Entdeckung eines MM, einer anderen Hautkrebsform oder einer Vorstufe von Hautkrebs dienen. In ähnlicher Weise werden erst ab dem 45 . Lebensjahr Männer zu solchen Hautver- änderungen befragt. Selbst- und/oder Partnerbeobachtung kann den Verdacht auf ein MM begründen. Masseure, Friseure, Fuß- und Handpfleger können ihre beruflichen Kontakte zur Beobachtung von und Aufklärung über Hautveränderungen nutzen. Dabei kann die ABCD-Regel hilfreich sein:

- A: Asymmetrie,

- B: unregelmäßige Begrenzung,

- C: Farbunregelmäßigkeiten,

- D: Durchmesser größer als $5 \mathrm{~mm}$.

Dazu können Dermatologen weitere Merkmale wie Erhabenheit, Störungen des Hautreliefs und andere Kriterien zur Abklärung eines solchen Verdachtes beurteilen. Mit dem einfachen Hilfsmittel der Auflichtmikroskopie kann die Sensitivität der Diagnose auf 90\% gesteigert werden. Dadurch gelingt es, ohne eingreifende Maßnahmen neun von zehn MM zu erkennen $[2,16]$.

\section{Fazit für die Praxis}

Im Verlauf der 80er Jahre nimmt die Tumordicke, der wichtigste Prognosefaktor, so deutlich ab, wie sich die Überlebensaussichten mit MM verbessern. Frauen weisen dabei die dünneren MM und die besseren Überlebensaussichten auf als Männer. Der Anstieg der Erkrankungsraten hat nicht zu einem Anstieg der Sterberaten der Frauen und nur zu einem geringeren Anstieg der Sterberaten der Männer geführt. Der Anstieg der Erkrankungsraten kann hier als Anzeichen einer frühzeitigeren Diagnose immer dünnerer und daher besser zu behandelnder MM gesehen werden. Das zeigen höhere altersspezifische Erkrankungsraten Jüngerer bei Frauen und Älterer bei Männern. Eine verbesserte Früherkennung sollte die Sterblichkeit an MM trotz des bei Männern später einsetzenden „gesetzlichen Früherkennungsprogramms" senken helfen. Die altersspezifischen Sterberaten der alten Bundesländer an MM folgen einem Muster, das in angelsächsischen Ländern als Geburtskohorteneffekt identifiziert wurde. Danach ist es beginnend mit den zwischen 1890 und 1905 Geborenen zu einem Anstieg des MM-Sterberisikos aufeinander folgender Geburtsjahrgänge gekommen, der erst mit den zwischen 1925 und 1935 Geborenen beendet ist. Jüngere Geburtsjahrgänge haben das gleiche oder ein geringeres MM-Risiko zu tra- 
gen wie die zwischen 1925 und $1935 \mathrm{Ge}$ borenen, die zur Zeit um 70 Jahre alt sind. In altersspezifischer Gliederung weisen daher nur noch die Sterberaten über 70-Jähriger bis zum Ende der 90 er Jahre eine Zunahme auf. Mit einer weiteren Zunahme der Sterberaten ist nicht zu rechnen. Im Gegenteil: Der Zusammenhang zwischen intensiver Sonnenexposition - Sonnenbaden und Sonnenbrand - in der Kindheit und der Entwicklung von Pigmentmalen, die das spätere MM-Risiko des Erwachsenen bestimmen, ermöglicht einfache Maßnahmen zur primären Prävention. Dazu gehört eine vorsichtigere, Sonnenbrände vermeidende Bräunung und ein Verzicht auf Bräunung, wenn dies der individuelle Hauttyp nicht zulässt. Die Kenntnis des eigenen Hauttyps und die Rücksichtnahme darauf ist wesentlicher Teil der Prävention des MM. Da sich auch aus Sonnenbränden im Erwachsenenalter ein mögliches MM-Risiko ergeben könnte, sind Vorsichtsmaßnahmen nicht auf die Kinder beschränkt und so leichter zu vermitteln. Eine sehr einfache und auch für Kinder umsetzbare Maßnahme zur Prävention des MM besteht in der Einhaltung einer Mittagsruhe im Schatten, wie die mediterranen Völker sie vorleben.

\section{Literatur}

1. Arbeitsgemeinschaft Bevölkerungsbezogener Krebsregister in Deutschland: Batzler WU, Schön D, Baumgart-Elms C, Schüz J, Eisinger B, Stegmeier C, Lenert M (1999) Krebs in Deutschland - Häufigkeiten und Trends. 2. aktualisierte Aufl. Eigenverlag, Saarbrücken

2. Arbeitsgemeinschaft Dermatologische Onkologie: Kaufmann R, Tilgen W, Garbe C (2001) Diagnostische und therapeutische Standards in der dermatologischen Onkologie: Malignes Melanom. ADO online

3. Breslow A (1970) Thickness, cross sectional areas and depth of invasion in the prognosis of cutaneous melanoma. Ann Surg 172:902-908
4. Clark WH, From L, Bernadino EA, Mihm MC (1969) The histogenesis and biological behavior of primary human malignant melanoma of the skin. Cancer Res 29:705-726

5. Balch CM, Soong Sj, Shaw HM, Milton GW (1992) An analysis of prognostic factors in 8500 patients with cutaneous melanoma. In: Balch CM, Houghton AN, Milton GW, Sober AJ, Soong $S$ (eds) Cutaneous melanoma. JB Lippincott, Philadelphia, pp 321-352

6. Garbe C, Büttner P, Bertz J et al. (1995) Primary cutaneous melanoma: identification of prognostic groups in 5093 patients and estimation of individual prognosis. Cancer 75:2484-2491

7. Armstrong BK, Krieger A (1994) Cutaneous melanoma. In: Cancer Surveys Volume 19. Trends in Cancer Incidence and Mortality, pp 219-240

8. Lee JAH, Petersen GR, Stevensen RG, Vesanen K (1979) The influence of age, year of birth, and date on mortality from malignant melanoma in the populations England and Wales, Canada, and the white population of the United States. Am J Epidemiol 110:734-739

9. Schön D, Bertz J, Görsch B et al. (1999) Entwicklung der Überlebensraten von Krebspatienten in Deutschland. Robert Koch-Institut, Berlin, S 141-147

10. Stroebel W, Garbe C, d'Hoedt B, Bertz J, Rassner G, Orfanos CE (1990) Klinisch-epidemiologische Daten des Zentralregisters Malignes Melanom der Deutschen Dermatologischen Gesellschaft. In: Orfanosa CE, Garbe C (Hrsg) Das maligne Melanom der Haut. W.Zuckerschwerdt, München, S 13-18

11. Marks R (1995) An overview of skin cancer. Cancer 75:607-612

12. Greene MH, Clark WH Jr, Tucker MA et al. (1985) Aquired precursors of cutaneous malignant melanoma: the familial dysplastic nevus syndrome. N Engl J Med 312:91-97

13. Elwood JM, Jopson J (1997) Melanoma and sun exposure: an overview of published studies. Int J Cancer 73:198-203

14. Autier P, Dore JF, Cattaruzza MS (1998) Sunscreen use, wearing clothes, and number of nevi in 6- to 7-year-old European children. J Natl Cancer Inst 90:1873-1880

15. Pfahlberg A, Schneider D, Kölmel K, Gefeller 0 (2000) UV-Exposition in der Kindheit und im Erwachsenenalter: Welche Lebensperiode beeinflusst das Melanomrisiko entscheidender? Soz Präventivmed 45:119-124

16. Koh HK, Geller AC, Miller DR, Lew RA (1995) The early detection of screening for melanoma, international Status. Cancer 75:674-683
Informationen zum Sonnenschutz

Die Broschüre "Auf in die Sonne - wie Sie sich und Ihr Kind vor UV-Strahlung schützen" richtet sich an interessierte Laien und informiert ohne Panikmache. Leicht verständlich werden darin Fragen behandelt wie: Was ist UV-Strahlung? Wann ist sie besonders intensiv? Welche weiteren biologischen Auswirkungen hat Sonnenlicht auf den Organismus? Warum reagiert Kinderhaut anders auf Sonnenlicht? Sonne und Sonnenallergie, Sonne und Krebs - wie hängt das zusammen?

Die kostenlose Broschüre ist gegen Einsendung eines mit drei Mark frankierten $\mathrm{C}_{5}$-Rückumschlags erhältlich beim Deutschen Grünen Kreuz e.V., "Auf in die Sonne", Schuhmarkt 4, 35037 Marburg. 